\title{
Gallbladder carcinoma associated with anomalous union of pancreatobiliary ductal system*
}

\author{
Sun Young Yi \\ Division of Gastroenterology, Internal Medicine, School of Medicine, Ewha Womans University, Seoul, South Korea \\ Email: syy@ewha.ac.kr
}

Received 11 June 2013; revised 16 July 2013; accepted 28 July 2013

Copyright (C) 2013 Sun Young Yi. This is an open access article distributed under the Creative Commons Attribution License, which permits unrestricted use, distribution, and reproduction in any medium, provided the original work is properly cited.

\begin{abstract}
A 64-year-old woman was admitted with mild right upper quadrant abdominal pain. Her physical examination revealed a moderately nourished female with normal vital sign and no evidence of jaundice or anemia. Preoperative diagnosis was choledochal cyst with anomalous union of pancreaticobiliary duct (AUPBD), long Y-shaped type and gallbladder (GB) cancer. An extended cholecystectomy, excision of the common bile duct and biliary diversion via Roux-en Y hepaticojejunostomy was performed. The gallbladder cancer showed invasion to perimuscular connective tissue and no extension beyond serosa. Twelve months after curative resection, the patient had no evidence of tumor recurrence.
\end{abstract}

Keywords: Choledochal Cyst; Gallbladder Cancer

\section{INTRODUCTION}

In anomalous union of pancreaticobiliary duct (AUPBD), the common channel is abnormally long and the connection between the common bile duct and pancreatic duct is outside of the duodenal wall [1]. This congenital disorder is frequently associated with choledochal cystst [1]. Moreover, AUPBD is prone to have benign as well as malignnant complications including carcinoma of biliary tract [2]. I describe here a case of a choledochal cyst combined with Y-shaped AUPBD that was detected in a patient in her sixties as a primary cancer of the gallbladder.

\section{CASE REPORT}

A 64-year-old woman was admitted with mild right upper quadrant abdominal pain. On abdominal physical examination, no palpable mass or tenderness was noted. Laboratory results revealed an elevated serum aspartate

\footnotetext{
${ }^{*}$ No conflicts of interest exist.
}

aminotransferase of $472 \mathrm{IU} / \mathrm{L}$, alanine aminotransferase of $240 \mathrm{IU} / \mathrm{L}$, alkaline phosphatase of $677 \mathrm{IU} / \mathrm{L}$ and total bilirubin of $4.0 \mathrm{mg} \%$. Serum carcinoembryonic antigen (CEA) was within normal limits and serum carbohydrate antigen 19-9 (CA19-9) level was slightly elevated (61.3 $\mathrm{IU} / \mathrm{L})$. Endoscopic ultrasound and abdominal computed tomography showed moderate dilated gallbladder with huge mass $(38 \mathrm{~mm})$ and marked dilated common bile duct (CBD) and abruptly narrowed far distal CBD (Figures 1 and 2). Endoscopic retrograde cholangiopancreatography (ERCP) and magnetic resonance cholangiopancreatogram (MRCP) revealed choledochal cyst with anomalous union of pancreatobiliary system (Figures $\mathbf{3}$ and 4). This patient presented with gallbladder mass and choledochal cyst associated with AUPBD, long Y-shaped type. An extended cholecystectomy, excision of the common bile duct and biliary diversion via Roux-en Y heapticojejunostomy was performed. On pathologic evaluation, the CBD was markedly dilated, measuring $3 \mathrm{~cm}$ in inner circumferences and $6 \mathrm{~cm}$ in length, and choledochal cyst type IA was revealed. Microscopic examination revealed papillay carcinoma of gallbladder, moderately differentiated and invades perimuscluar connective tissue without extension beyond (Figure 5). The patient's postoperative course was eventful, and she has had no clinical symptoms. Twelve months after curative resection, the patient had no evidence of tumor recurrence.

\section{DISCUSSION}

AUPBD is a congenital malformation of the pancreatobiliary tree in which the confluence of the common bile duct and pancreatic duct is outside the duodenal wall. The mode of anomalous union is classified into two types: the pancreatico-choledochal (P-C) type, in which the main pancreatic duct enters the common bile duct, and the C-P type, where the CBD enters the main pancreatic duct [1]. AUPBD has been regarded to be the etiologic factor of the choledochal cyst. However, choledochal cyst is not always associated with AUPBD. 


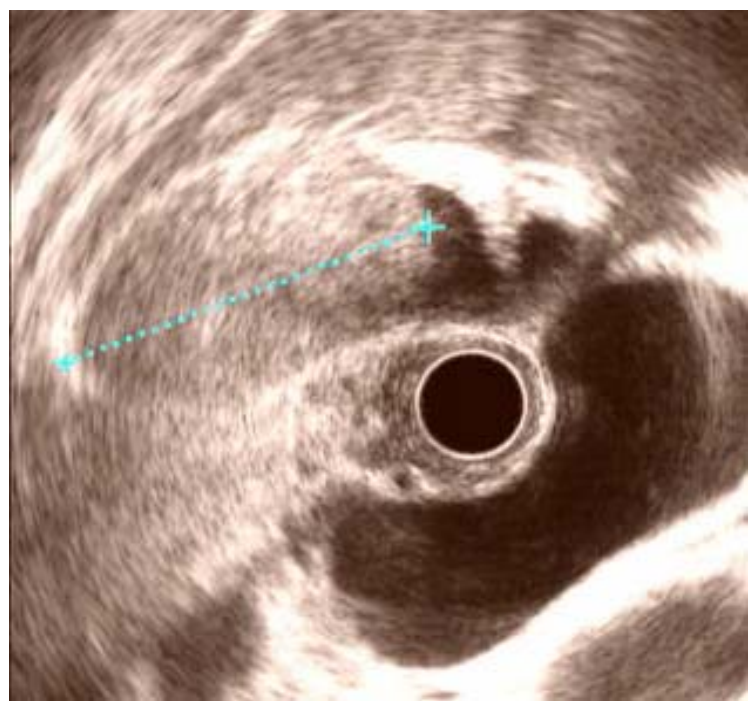

(a)

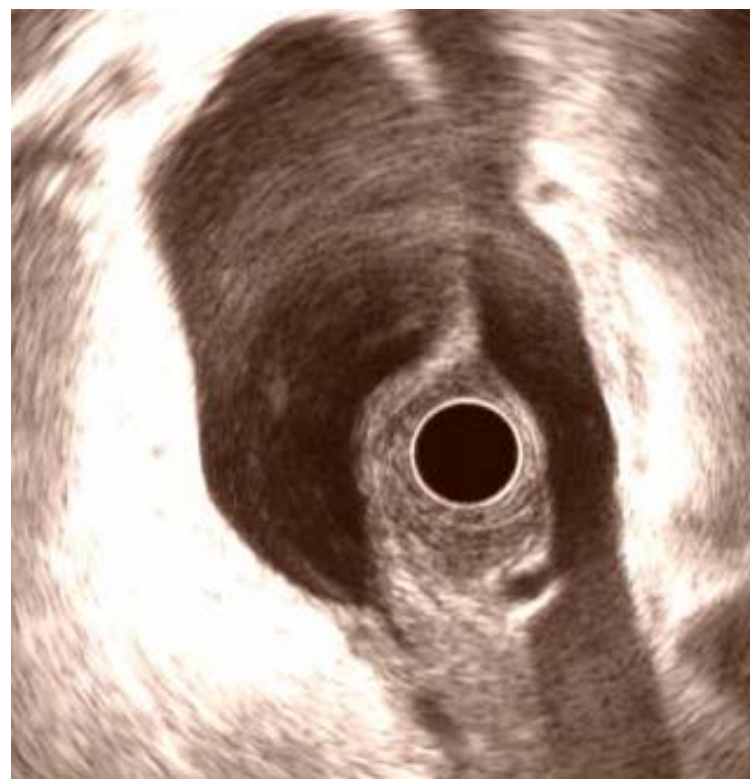

(b)

Figure 1. Endoscopic ultrasound showing huge mass (arrow) on gallbladder (a) and diffuse dilated common bile duct (arrow) (b).

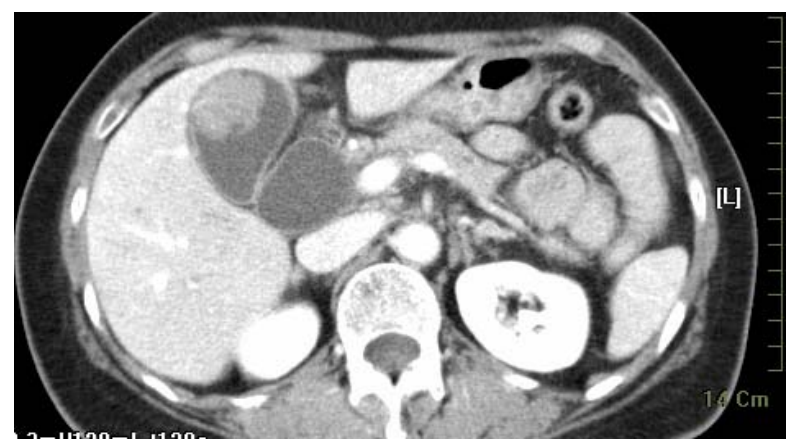

Figure 2. Abdominal scan showed polypoid mass of gallbladder (arrow) with dilated common bile duct.

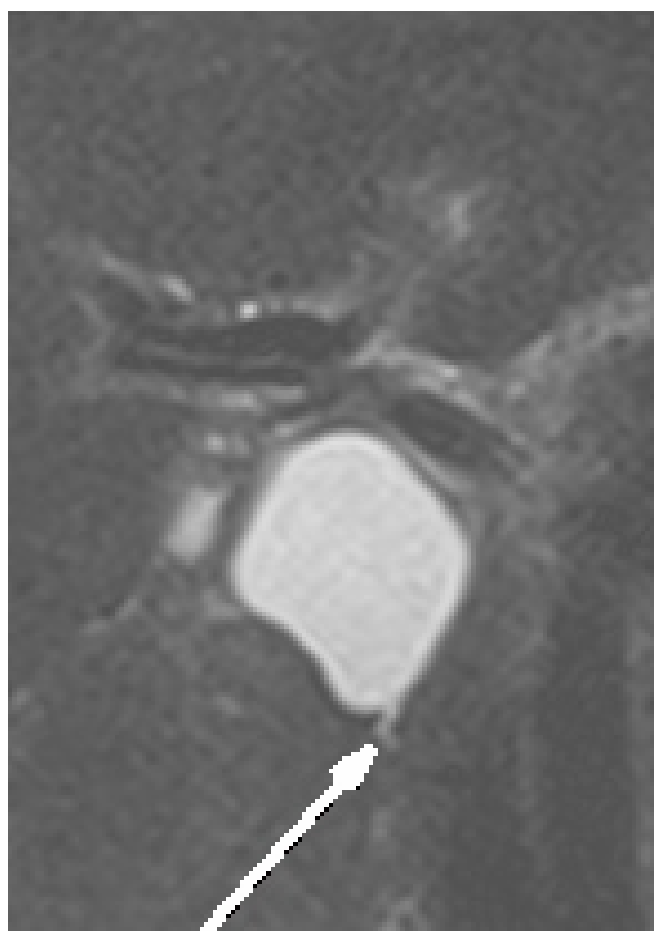

Figure 3. Magnetic resonance cholangiogram showed huge dilated common bile duct with abrupt narrowing distal bile duct (long arrow).

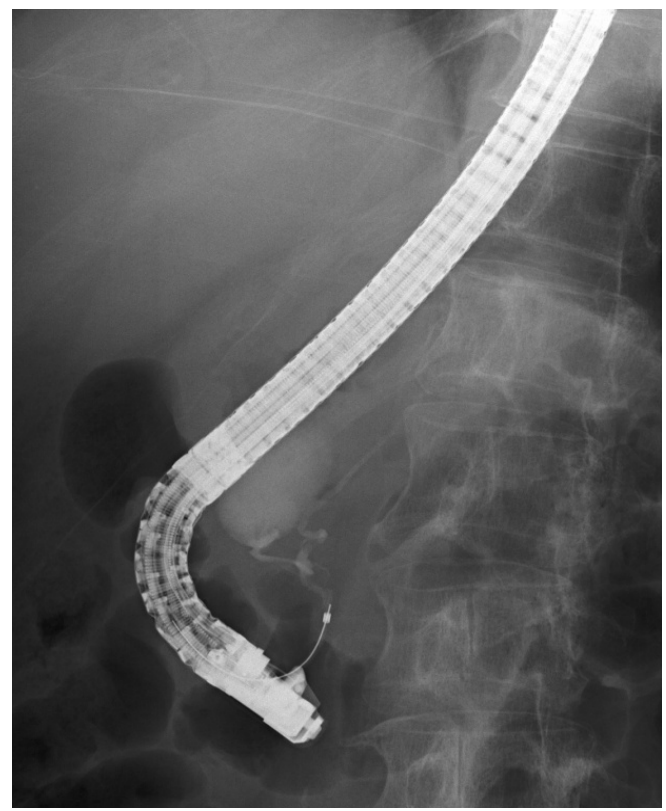

Figure 4. Endoscopic retrograde cholangiopancreatography (ERCP) demonstrated AUPBD with dilatation of the commo bile duct (long Y-shaped, arrow).

AUPBD associated with congenital bile duct dilation was $47.5 \%$, and that the incidence of congenital bile duct dilatation associated with AUPBD was $82.8 \%$ [2]. In one surgical series, $31 \%$ of patients with AUPBD presented 


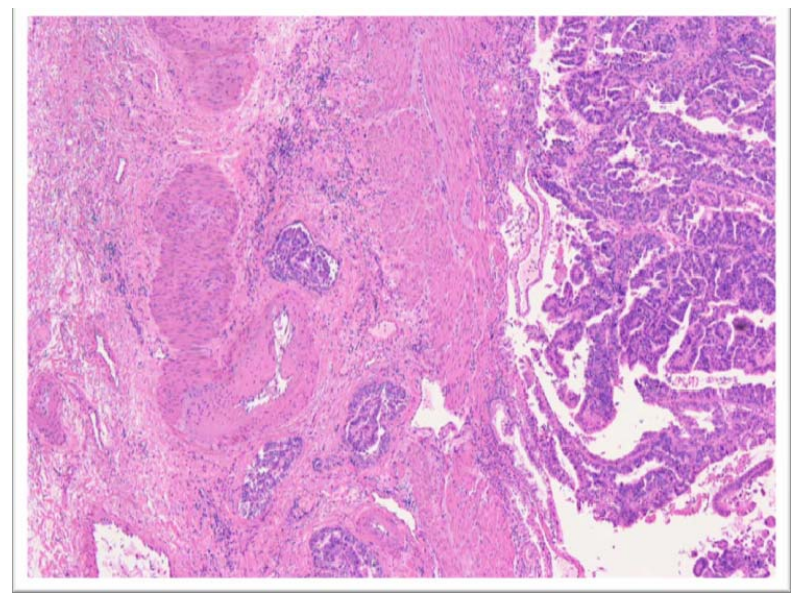

Figure 5. Hematoxylin \& Eosin stain image of tumor. Adenocarcinoma of the gallbladder, moderate differentiates and invades perimuscluar connective tissue without extension beyond $(\mathrm{H} \& \mathrm{E} ; \times 100)$.

with acute pancreatitis [3]. More often, it is the pancreatic juices that freely reflux into the bile duct, and this is believed to predispose patients to biliary cancer, primarily gallbladder adenocarcinoma (2.5\% to $26 \%)$ [4]. In fact, patients with an isolated AUPBD (without a choledochal cyst) may be at the highest risk for gallbladder cancer (up to 30\%) [5,6]. According to recent 10-year data from the Japanese Study Group on Pancreaticobiliary Maljunction, however, the rate of gallbladder cancer is higher than bile duct cancer with biliary dilatation [4]. The incidence of gallbladder cancer associated with AUPBD is reported to range from $16.7 \%$ to $18.3 \%$ [5].

This patient presented with gallbladder mass and choledochal cyst associated with AUPBD. An extended cholecystectomy, excision of the common bile duct and biliary diversion via Roux-en Y hepaticojejunostomy was performed. Microscopic examination revealed papillay carcinoma of gallbladder, moderate differentiated and invades perimuscluar connective tissue without extension beyond. And choledochal cyst type IA was revealed. The patient's post-operative course was eventful, and she has had no clinical symptoms.

\section{CONCLUSION}

Choledochal cysts and AUPBD are closely associated with biliary tract anomalies. The malignant potential of these disease entities usually requires early surgical intervention and lifelong follow-up.

\section{REFERENCES}

[1] The Japanese Study Group on Pancreaticobiliary Maljunction (JSPBM) (1994) The Committee of JSPBM for Diagnostic Criteria. Diagnostic criteria of pancreaticobiliary maljunction. Journal of Hepato-Biliary-Pancreatic Surgery, 1, 219-221. doi:10.1007/BF02391070

[2] Penn, E., Draganov, P. and Chauhan, S. (2010) Acute pancreatitis associated with anomalous pancreatobiliary junction. Clinical Gastroenterology and Hepatology, 8, 22. doi:10.1016/j.cgh.2010.05.005

[3] Sugiyama, M., Atomi, Y. and Kuroda, A. (1999) Pancreatic disorders associated with anomalous junction. Surgery, 126, 492-497. doi:10.1016/S0039-6060(99)70090-5

[4] Funabiki, T., Matsubara, T., Miyakawa, S. and Ishihara, S. (2009) Pancreaticobiliary maljunction and carcinogenesis to biliary and pancreatic malignancy. Langenbeck's Archives of Surgery, 394, 159-169. doi:10.1007/s00423-008-0336-0

[5] Sabdoh, N., Shirai, Y. and Hatakeyama, K. (1997) Incidence of anomalous union of the pancreatobiliary ductal system in biliary cancer. Hepatogastroenterology, 44, 1580-1583.

[6] Tashiro, S., Imaizumi, T., Ohkawa, H., Okada, A., Katoh, T., Kawaharada, Y., Shimada, H., Takamatsu, H., Miyake, H. and Todani, T. (2003) Pancreaticobiliary maljunction: Retrospective and nationwide survey in Japan. Journal of Hepato-Biliary-Pancreatic Surgery, 10, 345-351. doi:10.1007/s00534-002-0741-7 